\title{
Overview of current and future biologically based targeted therapies in head and neck squamous cell carcinoma
} Ajay Matta ${ }^{1}$ and Ranju Ralhan*1,2,3

\begin{abstract}
Address: ${ }^{1}$ Department of Chemistry and Centre for Research in Mass Spectrometry, York University, 4700 Keele Street, Toronto, Ontario, M3J 1P3 Canada , ${ }^{2}$ Joseph and Mildred Sonshine Family Centre for Head \& Neck Disease, Mount Sinai Hospital, 600 University Avenue, Toronto, Ontario, M5G 1X5, Canada and ${ }^{3}$ Department of Otolaryngology - Head and Neck Surgery, University of Toronto, 190 Elizabeth Street, Toronto, M5G 2N2, Ontario, Canada

Email: Ajay Matta - matta.ajay@gmail.com; Ranju Ralhan* - ralhanr@gmail.com

* Corresponding author

Published: 2 March 2009

Head \& Neck Oncology 2009, I:6 doi:10.1 186/1758-3284-I-6

This article is available from: http://www.headandneckoncology.org/content/l/I/6

(C) 2009 Matta and Ralhan; licensee BioMed Central Ltd.

This is an Open Access article distributed under the terms of the Creative Commons Attribution License (http://creativecommons.org/licenses/by/2.0), which permits unrestricted use, distribution, and reproduction in any medium, provided the original work is properly cited.
\end{abstract}

\begin{abstract}
Recent advances in genomics, proteomics, bioinformatics and systems biology have unraveled the complex aberrant signaling networks in cancer. The knowledge accrued has dramatically increased the opportunities for discovery of novel molecular targets for drug development. Major emphasis is being laid on designing new therapeutic strategies targeting multiple signaling pathways for more effective disease management. However, the translation of in vitro findings to patient management often poses major challenges that limit their clinical efficacy. Here we will discuss how understanding the dysregulated signaling networks can explain the pitfalls in translating the laboratory findings from the bench-to-bedside and suggest novel approaches to overcome these problems using head and neck cancer as a prototype. The five year survival rates of HNSCC patients (about $50 \%$ at 5 years) have not improved significantly despite advancements in multimodality therapy including surgery, radiation and chemotherapy. Molecular targeted therapies with inhibitors of EGFR and VEGF either alone, or in combination with conventional treatments have shown limited improved efficacy. The key deregulated signaling pathways in head and neck squamous cell carcinoma (HNSCC) include EGFR, Ras, TGF $\beta$, NFKB, Stat, Wnt/ $\beta$-catenin and PI3$\mathrm{K} / \mathrm{AKT} / \mathrm{mTOR}$. The aberrant activities of these interrelated signaling pathways contribute to HNSCC development. In depth understanding of the cross-talks between these pathways and networks will form the basis of developing novel strategies for targeting multiple molecular components for more effective prevention and treatment of HNSCC.
\end{abstract}

\section{Introduction}

Head and neck squamous cell carcinoma (HNSCC) is the sixth most common cancer accounting for over 500,000 new cases annually worldwide [1]. Despite improvement in treatment strategies involving surgery, radiotherapy (RT) and/or chemotherapy (CT), the prognosis of HNSCC patients in advanced stages (III/IV) remains largely unsat- isfactory owing to loco-regional recurrence $[2,3]$. Randomized trials using CT (cisplatin/carboplatin alone, or in combination with 5-Fluorouracil (5-FU), methotrexate or paclitaxel and/or RT show increased loco-regional control or survival and prevent subsequent metastasis by eradicating occult metastasis, though the dose limiting toxicities or increased risk of cardiac failure in cancer patients limits 
their clinical utility [4-6]. Hence major thrust is being laid on development of molecular targeted therapies for HNSCCs.

Multiple epigenetic and genetic events, including the aberrant expression and/or function of regulators of cell cycle, growth and signaling, motility, apoptosis, angiogenesis and microRNAs are implicated in pathogenesis of HNSCCs and constitute plausible targets for therapy. Advances in epigenomics, genomics, proteomics, bioinformatics and integration of this knowledge have provided holistic understanding of signaling pathways and networks that regulate cellular functions, intra- and intercellular communication, and tumor-host interactions. The deregulation of signaling cascades including the
EGFR, Ras, NFkB, Stat, Wnt/ $\beta$-catenin, TGF- $\beta$, and PI3-K/ AKT/mTOR pathways contributes to development of HNSCC [7]. Here, we will discuss how this emerging information on cross-talks between the different signaling pathways and networks can help to understand the limited efficacy of mono-targeted therapies for HNSCC. In turn, this knowledge can be harnessed for developing novel multiple molecular-targeted strategies for HNSCC treatment.

\section{Molecular Targeted Therapies for HNSCC}

Several molecular targeted therapies are currently being developed for HNSCC. The signaling pathways deregulated in HNSCC and the agents targeting key components are schematically represented in Figure 1 . The clinical effi-

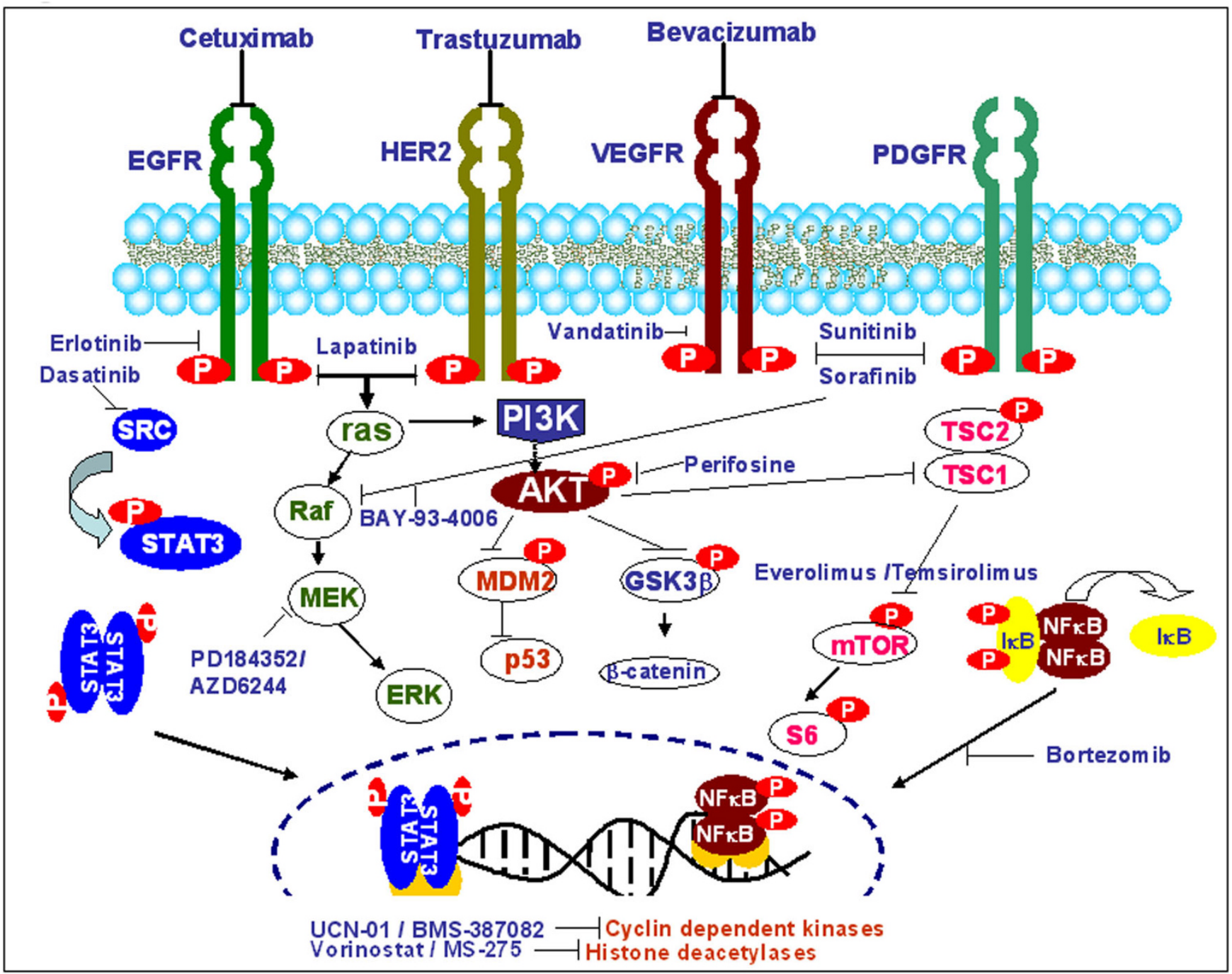

Figure I

Signaling pathways frequently deregulated in HNSCC, the molecular targets involved and their corresponding inhibitors as potential anticancer agents. 
cacies of these inhibitors targeting important pathways regulated by epidermal growth factor receptor (EGFR), vascular endothelial growth factor (VEGF) and AKT have been reviewed [8-14]. Large amount of preclinical in vitro and in vivo data have been obtained on the anti-proliferative properties of these inhibitors, both as single agents and combined with CT/RT. The inclusion of these agents in combined modality treatment regimes for early and/or advanced stage HNSCC is likely to increase therapeutic efficacy. Consequently, several targeted agents are under clinical trials in HNSCC, with many phase I/II studies already completed and some phase III studies in progress. The limited efficacies of these trials and unexpected toxicities in HNSCC patients have emphasized the difficulties of translating in-vitro findings to clinics for disease management.

\section{EGFR Inhibitors: Clinical Challenges}

Activation of EGFR signaling is one of the mechanisms for resistance to RT and/or CT in HNSCC, making it the most plausible therapeutic target [15-17]. Upon ligand binding (EGF or TGF $\alpha$ ), EGFR forms a homodimer or heterodimer with other members of the Erb family (Her2/ neu, Erb3, Erb4) and activates downstream signaling cascades-Ras/Raf/MAPK and the PI3K/Akt/mTOR pathways (Figure 1). The activation of these signaling events is responsible for regulating key tumorigenic processes such as proliferation, inhibition of apoptosis, cell adhesion/ motility, growth and survival. Monoclonal antibodies against the extra-cellular domain of EGFR, cetuximab, pertuzumab, panitumumab and trastuzumab, used as inhibitors in monotherapy have shown limited efficacy. In a phase I/II trial, combination of cetuximab with 5-FU and carboplatin/cisplatin showed increased survival with no cumulative toxicity in recurrent HNSCC [18]. Cetuximab acts as a tumor specific radiosensitizer $[19,20]$. EGFR inhibition by cetuximab significantly reduced tumor repopulation during fractionated RT in a xenografted human model of SCC [21]. In contrast, trials with chemoradiation (CRT) and cetuximab in HNSCC have shown adverse events and withdrawal of the trial [22]. Humanized antibodies- panitumumab and trastuzumab overcome the dose dependent toxicity of cetuximab but are less immunogenic and effective. Moreover, clinical trials of trastuzumab for HNSCC have reported cardiomyopathy in patients undergoing treatment. ErbB2 inhibition has been shown to activate mitochondrial apoptosis by modulating the ratio of BCL-xL and -xS, resulting in cardiomyopathy [23], but the exact mechanism of trastuzumab induced cardiomyopathy is still unclear. Unlike monoclonal antibodies, tyrosine kinase inhibitors (TKIs) such as gefitinib, erlotinib and lapatinib block the ATP pocket of EGFR, thereby inhibiting phosphorylation and downstream signal transduction. A multicentric study showed that erlotinib was well tolerated in pretreated HNSCCs and prolonged disease free survival [24].
Although, EGFR overexpression is observed in more than $90 \%$ of HNSCCs, yet, only a subset of these tumors show a clinically meaningful response to EGFR inhibition $[25,26]$. Potential reasons for failure of response to EGFR inhibitors include: constitutive activation of the Ras/Raf/ MAPK, STAT3 and PI3-K/AKT/mTOR signaling pathways independent of EGFR by other stimuli such as hypoxia, Ras activation or PTEN mutation and inhibition. The presence of EGFR variant III (EGFRvIII) in HNSCCs, is also responsible for constitutive activation of downstream signaling and resistance to EGFR inhibition by monoclonal antibodies [27]. Acquired resistance to cetuximab is accompanied by deregulation of EGFR internalization/ degradation and subsequent EGFR dependent activation of HER3 [28]. EGFR inhibition by erlotinib/gefitinib is overcome by epithelial-mesenchymal transition [29]. Recently, Hadad et al., [30] proposed a novel mechanism for regulation of mesenchymal phenotype and resistance to erlotinib in HNSCC cells by Delta-crystallin enhancer binding factor 1 . In addition, cross-talks between EGFR and cell adhesion molecules, cytokine receptors, ion channels and G protein coupled receptor (GPCR) lead to EGFR activation [31]. GPCR-EGFR cross-talk may play a role in development of HNSCC and account for limited efficacy of EGFR inhibitors in HNSCC. The aforementioned mechanisms might also explain why most clinical trials suggest no correlation between EGFR protein expression and response to EGFR inhibitors. Notably, favorable outcome has been associated with skin toxicity or presence of shorter EGFR intron 1 cytosine-adenine repeats [32]. As yet, there are no proven molecular predictors of response to EGFR targeted antibodies [33]; search for biomarkers should be extended to EGFR-activation status and key components of downstream pathways. Tumor signaling pathway components that work synergistically with EGFR or compensate for the loss of EGFR-initiated signaling are likely to be ideal targets for multi-targeted therapy. Erb family-targeted and Src family-targeted agents are in clinical development [34].

\section{VEGF Inhibitors}

Increased expression of VEGF and its receptors in HNSCCs, underscores the importance of VEGF pathway in angiogenesis and survival of tumor cells under hypoxic conditions [35,36]. VEGF expression is regulated by hypoxia-inducible factor $-1 \alpha$ (HIF-1 $\alpha$ ) -dependent and independent processes, both of which involve PI3-K and AKT. Bevacizumab, a humanized VEGF monoclonal antibody, not only inhibits angiogenesis, but also facilitates the increased delivery of chemotherapeutic agents by decreasing microvascular permeability and decreasing intratumor pressure. But, single-agent anti-angiogenic drugs have not shown activity in unselected HNSCC patients, with a response rate of less than $4 \%$. On the other hand, combinations of bevacizumab with erlotinib showed a response rate of $14.6 \%$. Studies of bevacizumab 
with CT (phase III Eastern Cooperative Oncology Group [ECOG] trial) and in combination with CRT are currently in progress [37]. However, HNSCCs show inter-tumoral angiogenic heterogeneity; in-depth understanding of the variability of angiogenic phenotype within a given HNSCC is important for designing cytokine targeted antiangiogenic therapies.

\section{Multikinase Inhibitors}

Sorafenib, an oral multikinase inhibitor, targets serine/ threonine Raf-1 kinase and receptor tyrosine kinases (RTKs)- VEGFR, PDGFR, KIT, and Flt3. Phase II trials in recurrent or metastatic HNSCC patients showed that sorafenib was well tolerated with modest anticancer activity comparable to monotherapy [38]. BIBF 1120, targets VEGF, PDGF, FGF receptor and src family of tyrosine kinases (Src, LcK, Lyn). Vandetanib (ZD6474), an inhibitor of VEGFR, EGFR, and rearranged during transfection (RET) tyrosine kinases is being tested in HNSCC as monotherapy and also in combination with CT $[39,40]$. Dasatinib (BMS-354825) is a synthetic, small molecule inhibitor of Src family kinases, also inhibits protein tyrosine kinases: bcr-abl, EphA2, PDGF- $\beta$ [41]. But, a major challenge in development of multikinase inhibitors is the rapid evolution of mutant inhibitor resistant kinases, therefore, appropriate multi-targeted inhibitors or combinations need to be planned in advance of clinical application.

\section{PI3-KIAKT/mTOR pathway Inhibitors}

Uncontrolled activation of the PI3-K/Akt/mTOR pathway contributes to the development and progression of HNSCC and is an important target to counteract resistance to RT and/or CT [42]. PTEN deletions and 'hot-spot' mutations of the PI3K gene have been shown to possess transforming capacity in vitro and in vivo, hence restoration of mutated or absent PTEN activity might be a target for AKT inhibition. Protease inhibitors downregulate the phosphorylation and expression of active PI3-K, that is responsible for radioresistance in HNSCC. Akt activation is a possible mechanism of resistance to EGFR inhibitors, therefore, the combination of AKT inhibitors and antiEGFR agents may be useful in effective management of HNSCC. The mammalian target of rapamycin, commonly known as mTOR regulates cell growth, proliferation, motility, survival, protein synthesis, and transcription. Rapamycin derivatives such as everolimus, temserolimus and deforolimus are potent inhibitors of mTOR and do not share the problems of poor solubility and chemical stability of rapamycin. A clinical trial using cisplatin and everolimus (RAD-001) in HNSCC is in progress [43]. However, not all HNSCCs have activated PI3-K/Akt/ mTOR pathway, hence molecular signatures need to be developed to define patients that may benefit from inhibitors of this pathway. Moreover, mTOR inhibition blocks the natural negative feedback on insulin-like growth factor-1 receptor (IGF-1R) signaling impinging on PI3-K. This results in increased PI3-K and Akt activation which could potentially counteract the inhibition of mTOR. Dual inhibition of both IGF-1R signaling or TKIs, and mTOR may result in a superior anti-proliferative effect over each single strategy in HNSCCs $[44,45]$. The major limitation of mTOR inhibitors in clinical trials is their dose limiting toxicity. Hence, natural products, such as curcumin, which hit multiple cellular targets including mTOR, in combination with mTOR inhibitors may reduce the toxic side effects and augment the clinical efficacy.

\section{Novel Biological Targeted Agents for HNSCC}

Biological agents, pemetrexed and enzastaurin, an oral protein kinase $\mathrm{C}$ beta (PKCbeta) inhibitor, are showing considerable promise and no unexpected toxicities in phase I trials in combination with cisplatin $[46,47]$. Aurora kinase A (AURKA) inhibitor and paclitaxel in combination are also under investigation for HNSCC management [48]. Among the new COX-2 inhibitors, Salvianolic acid B (Sal-B) has shown promise for HNSCC prevention and treatment [49]. Geldanamycin analogues have demonstrated potent inhibition of Hsp90 demonstrating significant anti-tumor activity in both cell culture and animal studies. Bortezomib inhibits activation of $\mathrm{NF} \kappa \mathrm{B}$ and sensitizes these cells to chemotherapy, radiation, or immunotherapy without added toxicities [50-53]. Histone deacetylases (HDACs) are enzymes that regulate the acetylation of histone proteins and non-histone proteins including p53, p21, NFкB. Altered expressions of HDACs have been reported in several human malignancies including head and neck cancer. Inhibitors of HDAC such as suberoylanilide hydroxamic acid have been shown to induce growth arrest, differentiation and promote apoptosis of HNSCC cell lines [54]. HDAC inhibitors target $\mathrm{NF} \kappa \mathrm{B}$ and also increase radiosensitivity and thus may be tried in future trials.

\section{Challenges in Molecular Targeted Therapies for HNSCC}

The complexity of the aberrant signaling in HNSCC (Figure 1 \&2) explains why interfering with only single steps in these pathways have not shown marked clinical response in HNSCC patients. HNSCC cells have the ability to exploit diverse signaling pathways for growth advantage, cell survival and evasion of apoptosis. In fact, some of these processes may even be facilitated by the use of selective targeted agents and warrant interference at different stages for reducing the tumor burden effectively. Further, different etiological factors and risk habits can result in distinct genetic and epigenetic alterations, which may trigger different signaling pathways that impact development and progression of HNSCCs. A proof of principle is the constitutive activation of Ras/Raf/MAPK pathway due to Ras mutations in the areca quid chewing oral cancers, 


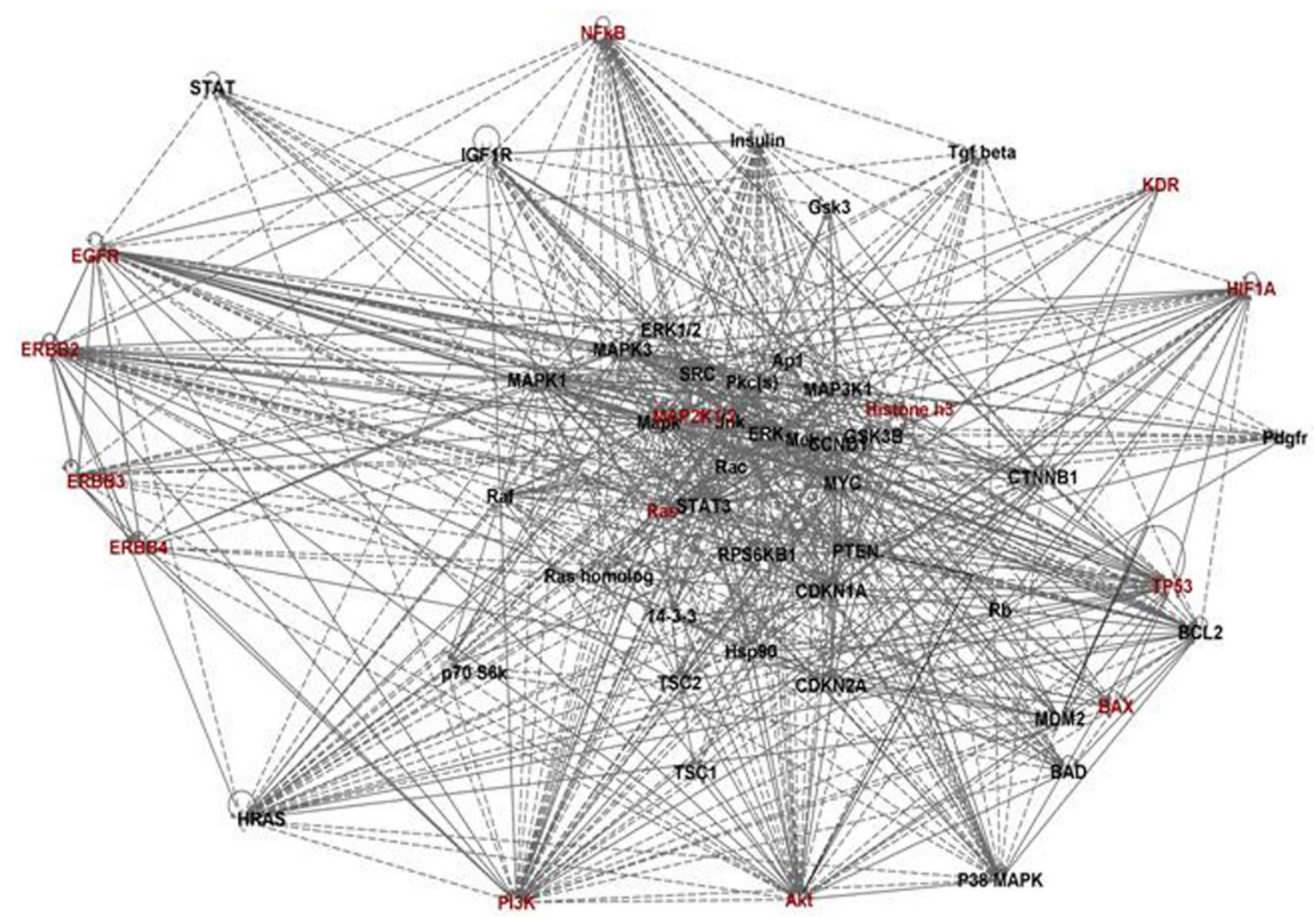

\section{Figure 2}

Network analysis using Ingenuity pathway analysis (IPA) software. This figure depicts the merged networks of proteins/molecules targeted for novel therapies in HNSCCs. These proteins/molecules form a complex network responsible for survival and proliferation of cancer cells. Therefore, targeting this complex network with single agents or monotherapy is often a failure as blocking a single pathway triggers alternate signaling cascades to activate downstream targets for survival. The bold lines show a direct association among different proteins/molecules while the dashed lines represent indirect regulation or association among these proteins. As shown, all members of Erb family are directly associated; hence dowregulating/blocking one of the members does not block downstream signaling via Ras/Raf/Mapk and PI3K/Akt/mTOR pathway completely. Also, as shown in figure, these pathways are also being regulated by Insulin, TGF- $\beta$ and KDR (VEGFR), PDGFR and HIFI $\alpha$.

while in cancers associated with chronic tobacco exposure this pathway is likely to be activated downstream from EGFR activation. Another evidence of multiple aberrant pathways is the altered NFKB function leading to activation of STAT3 by an autocrine or paracrine mechanism initiated by IL- 6 release, independent from EGFR. In addition, de novo/acquired chemoresistance comprises a significant problem in management of HNSCCs. The emerging data suggests Cancer Stem Cells (CSCs) may be responsible for acquired resistance to CT/RT in HNSCCs. Cancer stem cells is a subpopulation of cells that can selfrenew and produce differentiated cells that form the bulk of the tumor [55]. It is proposed that the current HNSCC treatment regimens selectively kills the differentiated can- cer cells producing tumor regression, but do not eliminate the cancer stem cells. Understanding the molecular signatures of HNSCC stem cells will define new targets for designing of novel therapeutic strategies.

Little attention has been paid to understanding the effect of human papillomavirus (HPV) on therapeutic response to targeted agents in HNSCC. It is being increasingly recognized that the molecular pathogenesis of HPV infected oropharyngeal SCC exhibits marked geographical variation [56] and is different from tobacco and alcohol associated HNSCC $[7,13,57]$, so how can these biologically different tumors show the same response to targeted agents? In fact, HPV associated HNSCC show better prog- 
nosis than HPV negative tumors, though the molecular basis of improved prognosis is not clearly understood. Nevertheless, in future trials, evaluation of HPV status of HNSCC patients is likely to provide better insight into the outcome of clinical response to targeted agents.

\section{Future Strategies}

The development of new biological agents should focus on inhibitors that are likely to hit multiple targets. Alternatively, combination of different agents that target distinct specific pathways is likely to inhibit the escape of tumor cells by alternate mechanisms leading to more effective disease control. But, the success of future clinical trials will depend upon (i) patient population and (ii) study design for assessment of response to therapy. Further, to evaluate the efficacy of these biological agents there is urgent need to identify novel biomarkers that can be used to accurately assess and individualize therapy.

\section{Patient population selection}

Phase II trials are often conducted on patients having advanced loco-regional disease or recurrent/metastatic HNSCC and have several limitations: (i) most patients have received $\mathrm{RT} / \mathrm{CT} / \mathrm{CRT}$ for the primary tumors, often develop multifactorial resistance and are less likely to respond to new agents effectively. This is best exemplified by the outcome of trials with cetuximab. In advanced HNSCC patients' refractory to platinum, cetuximab showed a response rate of $13 \%$, while the response rate increased to $20 \%$ in patients who were stable on platinum therapy [10]. Hence testing of new agents as first line therapy is likely to show better clinical response than in recurrent/metastatic HNSCC patients; (ii) cumulative resistance observed in recurrent/metastatic HNSCC patients limits the generalization of clinical response to patients with early disease for target validation; (iii) feasibility of conducting translational research is hampered by the ethical constraints in obtaining tumor biopsies. A paradigm shift in design of phase II trials is proposed that enables evaluation of new compounds in pre-operative window setting. The collection of biopsies before treatment at the time of diagnosis, and after treatment, either at the time of surgery or before loco-regional therapy will permit assessment of predictive molecular markers and may help in identifying subgroups of patients most likely to respond to therapy (or develop primary resistance). In addition, these paired tumor specimens are likely to provide insights into the pharmacodynamic effects of novel agents, and their mechanism of action. Establishment of a data base documenting the specificities of the inhibitors and observed toxicities in Phase I and Phase II studies would provide a valuable resource for understanding whether there are particular cellular targets whose inhibition should be avoided.

\section{Molecular markers for assessment of biological response}

Evaluation of clinical response dependent on progressionbased endpoints is likely to provide more realistic assessments of the anti-tumor activity of biological agents. Specific molecular markers may give a more objective evaluation of clinical response. To cite an example - pharmacodynamic tissue studies conducted on a phase I/II trial of erlotinib and cisplatin in patients with recurrent or metastatic HNSCC showed that high EGFR gene copy in tumor specimens may predict which patients are likely to respond to erlotinib, and decreased p-EGFR level in skin biopsies during therapy may represent a potential surrogate marker for improved clinical outcome. Multidimensional scaling (MDS) was shown to represent a novel way to evaluate these relationships between molecular markers and clinical outcome [58]. Molecular imaging or dynamic control enhanced CT/MRI may be used for measurement of intra-tumoral blood flow perfusion parameters to evaluate the clinical efficacy of anti-angiogenic agents.

There is a need for more predictive tumor models and better ways to monitor target inhibition in humans in a minimally invasive manner. As cell culture and animal models are severely limited in mimicking the development of human HNSCC, there is an urgent need to develop minimally invasive methods to discover and monitor biomarkers for evaluation of HNSCC in humans. New imaging modalities in conjunction with proteomic technologies may be investigated to monitor changes in signaling proteins and metabolites. Early detection technologies may allow us to diagnose and exterminate tumors prior to acquisition of survival capabilities that empower them with resistance to therapy.

In conclusion, in depth understanding of how the complex cellular signaling cascades and networks are reprogrammed in HNSCC and in the presence of monotargeting inhibitors is vital to rational designing of combinations of inhibitors. Innovative trial designs and appropriate patient selection are critical for the success of new trials to translate molecular targeted therapies from the bench to the clinics.

\section{Abbreviations}

5-FU: 5-Fluorouracil; ATP: Adenosine triphosphate; CRT: Chemoradiation; CSCs: Cancer Stem Cells; CT: Chemotherapy; ECOG: Eastern Cooperative Oncology Group; EGFR: Epidermal growth factor receptor; EGFRvIII: EGFR variant III; GPCR: G protein coupled receptor; HDACs: Histone deacetylases; HNSCCs: Head and Neck Squamous Cell Carcinomas; HPV: Human papillomavirus; IGF-1R: Insulin-like growth factor-1 receptor; PDGFR: Platelet derived growth factor receptor; RT: Radiotherapy; 
RTKs: Receptor tyrosine kinases; SCC: Squamous Cell Carcinoma; TKIs: Tyrosine kinase inhibitors; VEGF: Vascular endothelial growth factor; VEGFR: Vascular endothelial growth factor receptor.

\section{Competing interests}

The authors declare that they have no competing interests.

\section{Authors' contributions}

$\mathrm{AM}$ and RR wrote the manuscript. Both the authors read and approved the final manuscript.

\section{References}

I. Jemal A, Siegel R, Ward E, Hao Y, Xu J, Murray T, Thun MJ: Cancer statistics, 2008. CA Cancer J Clin 2008, 58:7I-96.

2. Pignon JP, le Maître A, Bourhis J, MACH-NC Collaborative Group: Meta-Analyses of Chemotherapy in Head and Neck Cancer (MACH-NC): an update. Int / Radiat Oncol Biol Phys 2007, 69(2 SuppI):SII2-SII4.

3. Scully C, Bagan JV: Recent advances in Oral Oncology 2007: imaging, treatment and treatment outcomes. Oral Oncol 2008, 44:2II-5.

4. Gibson MK, Li Y, Murphy B, Hussain MH, DeConti RC, Ensley J, Forastiere AA, Eastern Cooperative Oncology Group: Randomized phase III evaluation of cisplatin plus fluorouracil versus cisplatin plus paclitaxel in advanced head and neck cancer (EI395): an intergroup trial of the Eastern Cooperative Oncology Group. J Clin Oncol 2005, 23:3562-7.

5. Budach W, Hehr T, Budach V, Belka C, Dietz K: A meta-analysis of hyperfractionated and accelerated radiotherapy and combined chemotherapy and radiotherapy regimens in unresected locally advanced squamous cell carcinoma of the head and neck. BMC Cancer 2006, 6:28.

6. de Castro G Jr, Snitcovsky IM, Gebrim EM, Leitão GM, Nadalin W, Ferraz AR, Federico MH: High-dose cisplatin concurrent to conventionally delivered radiotherapy is associated with unacceptable toxicity in unresectable, non-metastatic stage IV head and neck squamous cell carcinoma. Eur Arch Otorhinolaryngol 2007, 264: 1475-82.

7. Molinolo AA, Amornphimoltham P, Squarize $\mathrm{CH}$, Castilho RM, Pate V, Gutkind JS: Dysregulated molecular networks in head and neck carcinogenesis. Oral Oncol 2008 in press.

8. Choong NW, Cohen EE: Epidermal growth factor receptor directed therapy in head and neck cancer. Crit Rev Oncol Hematol 2006, 57:25-43.

9. Harari PM, Huang S: Radiation combined with EGFR signal inhibitors: head and neck cancer focus. Semin Radiat Oncol 2006, 16:38-44.

10. Le Tourneau C, Faivre S, Siu LL: Molecular targeted therapy of head and neck cancer: review and clinical development challenges. Eur / Cancer 2007, 43:2457-66.

II. Shirai K, O'Brien PE: Molecular targets in squamous cell carcinoma of the head and neck. Curr Treat Options Oncol 2007, 8:239-5।.

12. Dietz A, Boehm A, Mozet C, Wichmann G, Giannis A: Current aspects of targeted therapy in head and neck tumors. Eur Arch Otorhinolaryngol 2008, 265 SuppI I:S3-SI 2.

13. Glazer CA, Chang SS, Ha PK, Califano JA: Applying the molecular biology and epigenetics of head and neck cancer in everyday clinical practice. Oral Oncol 2008 in press.

14. Langer C): Targeted therapy in head and neck cancer: state of the art 2007 and review of clinical applications. Cancer 2008, I I 2:2635-45.

15. Ang KK, Andratschke NH, Milas L: Epidermal growth factor receptor and response of head-and-neck carcinoma to therapy. Int J Radiat Oncol Biol Phys 2004, 58:959-65.

16. Rodemann HP, Dittmann K, Toulany M: Radiation-induced EGFRsignaling and control of DNA-damage repair. Int J Radiat Biol 2007, 83:781-91.
17. Kim S, Grandis JR, Rinaldo A, Takes RP, Ferlito A: Emerging perspectives in epidermal growth factor receptor targeting in head and neck cancer. Head Neck 2008, 30:667-74.

18. Bourhis J, Rivera F, Mesia R, Awada A, Geoffrois L, Borel C, Humblet Y, Lopez-Pousa A, Hitt R, Vega Villegas ME, Duck L, Rosine D, Amellal $\mathrm{N}$, Schueler A, Harstrick A: Phase I/II study of cetuximab in combination with cisplatin or carboplatin and fluorouracil in patients with recurrent or metastatic squamous cell carcinoma of the head and neck. J Clin Oncol 2006, 24:2866-72.

19. Bernier J: Drug Insight: cetuximab in the treatment of recurrent and metastatic squamous cell carcinoma of the head and neck. Nat Clin Pract Oncol 2008, 5:705-13.

20. Vermorken JB, Mesia R, Rivera F, Remenar E, Kawecki A, Rottey S, Erfan J, Zabolotnyy D, Kienzer HR, Cupissol D, Peyrade F, Benasso M, Vynnychenko I, De Raucourt D, Bokemeyer C, Schueler A, Amella $\mathrm{N}$, Hitt R: Platinum-based chemotherapy plus cetuximab in head and neck cancer. N Engl J Med 2008, 359: I I |6-27.

21. Teoh DC, Rodger S, Say J, Hartley A: Hypofractionated radiotherapy plus cetuximab in locally advanced head and neck cancer. Clin Oncol (R Coll Radiol) 2008, 20:7I7.

22. Pfister DG, Su YB, Kraus DH, Wolden SL, Lis E, Aliff TB, Zahalsky AJ, Lake S, Needle MN, Shaha AR, Shah JP, Zelefsky MJ: Concurrent cetuximab, cisplatin, and concomitant boost radiotherapy for locoregionally advanced, squamous cell head and neck cancer: a pilot phase II study of a new combined-modality paradigm. J Clin Oncol 2006, 24:072-8.

23. Guglin M, Cutro R, Mishkin JD: Trastuzumab-induced cardiomyopathy. J Card Fail 2008, I 4:437-44.

24. Soulieres D, Senzer NN, Vokes EE, Hidalgo M, Agarwala SS, Siu LL: Multicenter phase II study of erlotinib, an oral epidermal growth factor receptor tyrosine kinase inhibitor, in patients with recurrent or metastatic squamous cell cancer of the head and neck. J Clin Oncol 2004, 22:77-85.

25. Rogers SJ, Harrington KJ, Rhys-Evans P, O-Charoenrat P, Eccles SA Biological significance of c-erbB family oncogenes in head and neck cancer. Cancer Metastasis Rev 2005, 24:47-69.

26. Morgillo F, Bareschino MA, Bianco R, Tortora G, Ciardiello F: Primary and acquired resistance to anti-EGFR targeted drugs in cancer therapy. Differentiation 2007, 75:788-99.

27. Sok JC, Coppelli FM, Thomas SM, Lango MN, Xi S, Hunt JL, Freilino ML, Graner MW, Wikstrand CJ, Bigner DD, Gooding WE, Furnari FB, Grandis JR: Mutant epidermal growth factor receptor (EGFRvIII) contributes to head and neck cancer growth and resistance to EGFR targeting. Clin Cancer Res 2006, I 2:5064-73.

28. Eriala K, Sundvall M, Junttila TT, Zhang N, Savisalo M, Mali P, Kulmala J, Pulkkinen J, Grenman R, Elenius K: Signaling via ErbB2 and ErbB3 associates with resistance and epidermal growth factor receptor (EGFR) amplification with sensitivity to EGFR inhibitor gefitinib in head and neck squamous cell carcinoma cells. Clin Cancer Res 2006, I2:4103-II.

29. Frederick BA, Helfrich BA, Coldren CD, Zheng D, Chan D, Bunn PA $J r$, Raben D: Epithelial to mesenchymal transition predicts gefitinib resistance in cell lines of head and neck squamous cell carcinoma and non-small cell lung carcinoma. Mol Cancer Ther 2007, 6: |683-9|.

30. Haddad Y, Choi W, McConkey DJ: Delta-crystallin enhancer binding factor I controls the epithelial to mesenchymal transition phenotype and resistance to the epidermal growth factor receptor inhibitor erlotinib in human head and neck squamous cell carcinoma lines. Clin Cancer Res 2009, 15:532-42.

31. Zandi R, Larsen AB, Andersen P, Stockhausen MT, Poulsen HS: Mechanisms for oncogenic activation of the epidermal growth factor receptor. Cell Signal 2007, 19:2013-23.

32. Schwentner I, Witsch-Baumgartner M, Sprinzl GM, Krugmann J, Tzankov A, Jank S, Zwierzina $H$, Loeffler-Ragg J: Identification of the rare EGFR mutation p.G796S as somatic and germline mutation in white patients with squamous cell carcinoma of the head and neck. Head Neck 2008, 30:1040-4.

33. Krause $M$, Baumann $M$ : Clinical biomarkers of kinase activity: examples from EGFR inhibition trials. Cancer Metastasis Rev 2008, 27:387-402.

34. Egloff AM, Grandis JR: Targeting epidermal growth factor receptor and SRC pathways in head and neck cancer. Semin Oncol 2008, 35:286-97. 
35. Shang ZJ, Li ZB, Li JR: VEGF is up-regulated by hypoxic stimulation and related to tumour angiogenesis and severity of disease in oral squamous cell carcinoma: in vitro and in vivo studies. Int J Oral Maxillofac Surg 2006, 35:533-8.

36. Liang X, Yang D, Hu J, Hao X, Gao J, Mao Z: Hypoxia inducible factor-alpha expression correlates with vascular endothelial growth factor-C expression and lymphangiogenesis/angiogenesis in oral squamous cell carcinoma. Anticancer Res 2008, 28:1659-66.

37. Fujita K, Sano D, Kimura M, Yamashita $Y$, Kawakami M, Ishiguro $Y$, Nishimura G, Matsuda H, Tsukuda M: Anti-tumor effects of bevacizumab in combination with paclitaxel on head and neck squamous cell carcinoma. Oncol Rep 2007, I 8:47-5I.

38. Elser C, Siu LL, Winquist E, Agulnik M, Pond GR, Chin SF, Francis $P$, Cheiken R, Elting J, McNabola A, Wilkie D, Petrenciuc O, Chen EX: Phase II trial of sorafenib in patients with recurrent or metastatic squamous cell carcinoma of the head and neck or nasopharyngeal carcinoma. J Clin Oncol 2007, 25:3766-73.

39. Sano D, Kawakami M, Fujita K, Kimura M, Yamashita Y, Ishiguro $Y$, Nishimura G, Matsuda H, Tsukuda M: Antitumor effects of ZD6474 on head and neck squamous cell carcinoma. Oncol Rep 2007, 17:289-95.

40. Gustafson DL, Frederick B, Merz AL, Raben D: Dose scheduling of the dual VEGFR and EGFR tyrosine kinase inhibitor vandetanib (ZD Zactima) in combination with radiotherapy in EGFR-positive and EGFR-null human head and neck tumor xenografts. Cancer Chemother Pharmacol 6474, 61:179-88.

4I. Johnson FM, Saigal B, Talpaz M, Donato NJ: Dasatinib (BMS354825) tyrosine kinase inhibitor suppresses invasion and induces cell cycle arrest and apoptosis of head and neck squamous cell carcinoma and non-small cell lung cancer cells. Clin Cancer Res 2005, I I:6924-32.

42. Bussink J, Kogel AJ van der, Kaanders JH: Activation of the PI3-K/ AKT pathway and implications for radioresistance mechanisms in head and neck cancer. Lancet Oncol 2008, 9:288-96.

43. Nathan CO, Amirghahari N, Rong X, Giordano T, Sibley D, Nordberg M, Glass J, Agarwal A, Caldito G: Mammalian target of rapamycin inhibitors as possible adjuvant therapy for microscopic residual disease in head and neck squamous cell cancer. Cancer Res 2007, 67:2160-8.

44. Oh SH, Kim WY, Kim JH, Younes MN, El-Naggar AK, Myers JN, Kies $M$, Cohen P, Khuri F, Hong WK, Lee HY: Identification of insulinlike growth factor binding protein-3 as a farnesyl transferase inhibitor SCH66336-induced negative regulator of angiogenesis in head and neck squamous cell carcinoma. Clin Cancer Res 2006, | 2:653-61.

45. Jimeno A, Kulesza P, Wheelhouse J, Chan A, Zhang X, Kincaid E, Chen R, Clark DP, Forastiere A, Hidalgo M: Dual EGFR and mTOR targeting in squamous cell carcinoma models, and development of early markers of efficacy. Br J Cancer 2007, 96:952-9.

46. Carducci MA, Musib L, Kies MS, Pili R, Truong M, Brahmer JR, Cole $P$, Sullivan R, Riddle J, Schmidt J, Enas N, Sinha V, Thornton DE, Herbst RS: Phase I dose escalation and pharmacokinetic study of enzastaurin, an oral protein kinase $C$ beta inhibitor, in patients with advanced cancer. J Clin Oncol 2006, 24:4092-9.

47. Specenier PM, Ciuleanu T, Latz JE, Musib LC, Darstein CL, Vermorken JB: Pharmacokinetic evaluation of platinum derived from cisplatin administered alone and with pemetrexed in head and neck cancer patients. Cancer Chemother Pharmacol 2008 in press.

48. Mazumdar A, Henderson YC, El-Naggar AK, Sen S, Clayman GL: Aurora kinase $\mathrm{A}$ inhibition and paclitaxel as targeted combination therapy for head and neck squamous cell carcinoma. Head Neck 2008 in press.

49. Hao Y, Xie T, Korotcov A, Zhou Y, Pang X, Shan L, Ji H, Sridhar R, Wang P, Califano J, Gu X: Salvianolic acid B inhibits growth of head and neck squamous cell carcinoma in vitro and in vivovia cyclooxygenase-2 and apoptotic pathways. Int J Cancer 2008 in press.

50. Allen C, Saigal K, Nottingham L, Arun P, Chen Z, Van Waes C: Bortezomib-induced apoptosis with limited clinical response is accompanied by inhibition of canonical but not alternative nuclear factor-\{kappa\}B subunits in head and neck cancer. Clin Cancer Res 2008, 14:4175-85.
51. Chen Z, Ricker JL, Malhotra PS, Nottingham L, Bagain L, Lee TL, Yeh NT, Van Waes C: Differential bortezomib sensitivity in head and neck cancer lines corresponds to proteasome, nuclear factor-kappaB and activator protein-I related mechanisms. Mol Cancer Ther 2008, 7:1949-60.

52. $\mathrm{Li} \mathrm{C}, \mathrm{Li}$ R, Grandis JR, Johnson DE: Bortezomib induces apoptosis via Bim and Bik up-regulation and synergizes with cisplatin in the killing of head and neck squamous cell carcinoma cells. Mol Cancer Ther 2008, 7:1647-55.

53. Wagenblast J, Baghi M, Arnoldner C, Bisdas S, Gstöttner W, Ackermann H, May A, Knecht R, Hambek M: Effect of bortezomib and cetuximab in EGF-stimulated HNSCC. Anticancer Res 2008, 28:2239-43.

54. Gillenwater AM, Zhong M, Lotan R: Histone deacetylase inhibitor suberoylanilide hydroxamic acid induces apoptosis through both mitochondrial and Fas(Cd95) signaling in head and neck squamous carcinoma cells. Mol Cancer Ther 2007, 6:2967-75.

55. Prince ME, Ailles LE: Cancer stem cells in head and neck squamous cell cancer. J Clin Oncol 2008, 26:287I-5.

56. Herrero R, Castellsagué X, Pawlita M, Lissowska J, Kee F, Balaram P, Rajkumar T, Sridhar H, Rose B, Pintos J, Fernández L, Idris A, Sánchez M], Nieto A, Talamini R, Tavani A, Bosch FX, Reidel U, Snijders PJ, Meijer CJ, Viscidi R, Muñoz N, Franceschi S: IARC Multicenter Oral Cancer Study Group. J Natl Cancer Inst 2003, 95: 1772-83.

57. Lo WY, Lai $\mathrm{CC}$, Hua CH, Tsai MH, Huang SY, Tsai $\mathrm{CH}$, Tsai F]: SI00A8 is identified as a biomarker of HPVI8-infected oral squamous cell carcinomas by suppression subtraction hybridization, clinical proteomics analysis, and immunohistochemistry staining. J Proteome Res 2007, 6:2143-5I.

58. Agulnik M, da Cunha Santos G, Hedley D, Nicklee T, Dos Reis PP, Ho J, Pond GR, Chen H, Chen S, Shyr Y, Winquist E, Soulieres D, Chen EX, Squire JA, Marrano P, Kamel-Reid S, Dancey J, Siu LL, Tsao MS: Predictive and pharmacodynamic biomarker studies in tumor and skin tissue samples of patients with recurrent or metastatic squamous cell carcinoma of the head and neck treated with erlotinib. J Clin Oncol 2007, 25:2184-90.
Publish with Biomed Central and every scientist can read your work free of charge

"BioMed Central will be the most significant development for disseminating the results of biomedical research in our lifetime. "

Sir Paul Nurse, Cancer Research UK

Your research papers will be:

- available free of charge to the entire biomedical community

- peer reviewed and published immediately upon acceptance

- cited in PubMed and archived on PubMed Central

- yours - you keep the copyright
BioMedcentral 